\title{
The adenine nucleotide translocator 1 acts as a type 2 transglutaminase substrate: implications for mitochondrial-dependent apoptosis
}

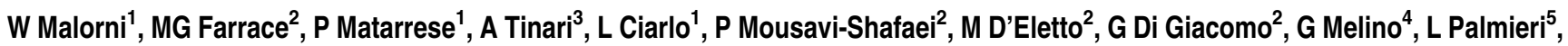 \\ C Rodolfo ${ }^{2}$ and M Piacentini ${ }^{\star 2,2,6}$
}

In this study we provide in vitro and in vivo evidence showing that the protein disulphide isomerase (PDI) activity of type 2 transglutaminase (TG2) regulates the correct assembly and function of the mitochondrial ADP/ATP transporter adenine nucleotide translocator 1 (ANT1). We demonstrate, by means of biochemical and morphological analyses, that ANT1 and TG2 physically interact in the mitochondria. Under physiological conditions, TG2's PDI activity regulates the ADP/ATP transporter function by controlling the oligomerization of ANT1. In fact, mitochondria isolated from hearts of TG2 ${ }^{-I-}$ mice exhibit increased polymerization of ANT1, paralleled by an enhanced ADP/ATP carrier activity, as compared to mitochondria belonging to TG2 ${ }^{+I+}$ mice. Interestingly, upon cell-death induction, ANT1 becomes a substrate for TG2's cross-linking activity and the lack of TG2 results in a reduction of apoptosis as well as in a marked sensitivity to the ADP/ATP exchange inhibition by atractyloside. These findings suggest a complex TG2-dependent regulation of the ADP/ATP transporter and reveal new important avenues for its potential applications in the treatment of some mitochondrial-dependent diseases, including cardiovascular and neurodegenerative diseases.

Cell Death and Differentiation (2009) 16, 1480-1492; doi:10.1038/cdd.2009.100; published online 31 July 2009

Transglutaminases (EC 2.3.2.13; TGases) are a group of $\mathrm{Ca}^{2+}$-dependent enzymes that catalyse the post-translational modification of proteins, through the incorporation of primary amines into the $\gamma$-carboxamide group of glutamine residues or by the cross-linking of proteins via $\varepsilon$ - $(\gamma$-glutamyl)lysine bridges. ${ }^{1}$ These cross-linked proteins become more resistant to mechanical, chemical and proteolytic injury. ${ }^{2}$

The most widely distributed transglutaminase isoenzyme is type 2 transglutaminase (TG2), which has been reported as a multifunctional protein, able to carry out a large number of biochemical functions. ${ }^{1,3}$ In the presence of high calcium levels, TG2 catalyses a vast array of protein post-translational modifications, including protein-protein cross-linkings, incorporation of primary amine into proteins as well as glutamine deamidation. In addition, TG2 might also act as a G-protein, coupling various receptors to phospholipase C (PLC $\delta 1) .{ }^{4}$ This activity is inhibited by $\mathrm{Ca}^{2+}$, which then acts as a switch between the two main biochemical functions of the enzyme. ., $^{5}$ Recently, a $\mathrm{Ca}^{2+}$-independent protein disulphide isomerase
$(\mathrm{PDI})^{7}$ and a kinase activity have been reported, ${ }^{8}$ further emphasizing the multifunctional nature of the protein.

Although it is now clear that TG2 possesses multiple and unique cellular features, mice bearing the deletion of the TG2 gene do not exhibit embryonic abnormalities. ${ }^{9}$ They are viable, born with mendelian frequency and do not show any evident abnormality, under normal physiological conditions. However, when cell death is induced, $\mathrm{TG}^{-1-}$ mice result to be defective in the in vivo clearance of apoptotic cells by phagocytosis both in the thymus and in the liver. Moreover, inflammatory as well as autoimmune reactions have been reported to develop spontaneously with age. ${ }^{10,11}$ In addition, $\mathrm{TG} 2^{-1-}$ mice show impaired insulin secretion with consequent glucose intolerance and hyperglycaemia, a phenotype strongly resembling a subtype of human diabetes named MODY (for maturity-onset diabetes of the young). ${ }^{12}$ Interestingly, in individuals with type 2 diabetes, $\beta$ cells do not sense glucose properly and therefore do not release appropriate amounts of insulin. ${ }^{13}$ Glucose sensing requires oxidative

'Department of Therapeutic Research and Medicine Evaluation, National Institute of Health, Rome, Italy; 'Department of Biology, University of Rome 'Tor Vergata', Rome, Italy; ${ }^{3}$ Department of Technology and Health, National Institute of Health, Rome, Italy; ${ }^{4}$ Department of Experimental Medicine and Biochemical Sciences, Biochemistry IDI-IRCCS Laboratory, University of Rome 'Tor Vergata', Rome, Italy; ${ }^{5}$ Department of Pharmaco-Biology, Laboratory of Biochemistry and Molecular Biology, University of Bari and CNR Institute of Biomembranes and Bioenergetics, Bari, Italy and ${ }^{6}$ National Institute for Infectious Diseases-IRCCS 'Lazzaro Spallanzani', Rome, Italy

${ }^{*}$ Correspondence: M Piacentini, Dipartimento di Biologia, Università di Roma 'Tor Vergata', Via della Ricerca Scientifica 1, 00133 Roma, Italia.

Tel: + 390672594234, E-mail: mauro.piacentini@uniroma2.it

Keywords: adenine nucleotide translocator; mitochondria; transglutaminase

Abbreviations: ANT1, adenine nucleotide translocator 1; ATRL, atractyloside; BA, bongkrekic acid; CyA, cyclosporine A; CyP-D, cyclophilin D; DDG, deoxy-D-glucose; EZ-link, 5-(biotinamido)pentylamine; IMM, inner mitochondrial membrane; IVM, intensified video microscopy; JC-1, 5-5',6-6'-tetrachloro-1,1',3,3'-tetraethylbenzimidazol-carbocyanine iodide; $\mathrm{MPP}^{+}, 1$-methyl-4-phenyl-pyridinium ion; K/S test, Kolmogorov-Smirnov test; $\Delta \psi \mathrm{m}$, mitochondrial membrane potential; MMP, mitochondrial membrane potential; MAb, monoclonal antibody; MODY, maturity-onset diabetes of the young; MEF, mouse embryonic fibroblast; 3-NP, 3-nitropropionic acid; OXPHOS, oxidative phosphorylation; PAb, polyclonal antibody; PDI, protein disulphide isomerase; PI, presidium iodide; PLC $\delta 1$, phospholipase C; ROS, reactive oxygen species; S.D., standard deviation; STS, staurosporine; TG2, type 2 transglutaminase; TMRM, tetramethylrhodamine ester; VDAC, voltage-dependent anion channel; WCE, whole cell extracts

Received 27.10.08; revised 29.5.09; accepted 03.6.09; Edited by SJ Martin; published online 31.7.09 
mitochondrial metabolism, leading to the generation of ATP. ${ }^{14}$ Recently, we showed that TG $2^{-1-}$ cardiomyocytes result more sensitive to ischemia/reperfusion injury than their wild-type counterparts. ${ }^{15}$ In addition, we observed a significant decrease in the levels of adenine nucleotide in the hearts of TG2 ${ }^{-1-}$ mice, as the result of an impaired mitochondrial function. ${ }^{16}$ These results highlighted an unexpected role for TG2 in the modulation of mitochondrial physiology. In fact, $\mathrm{TG} 2^{-1-}$ mice show a constitutive defect in the activity of mitochondrial respiratory complex $\mathrm{I}$, which is partially compensated by an increased activity of complex II. These features result in the protection of nigrostriatal neurons against damages induced by the complex I inhibitor, 1-methyl-4-phenyl-pyridinium ion $\left(\mathrm{MPP}^{+}\right)$. On the other side, $\mathrm{TG} 2^{-/-}$mice result more vulnerable to damages induced by methamphetamine or by the complex II inhibitor, 3-nitropropionic acid (3-NP). ${ }^{17}$

Under physiological conditions, the inner mitochondrial membrane (IMM) is impermeable to almost all metabolites and ions, and the permeability transition pore (PTP) is in a closed conformation, which is essential for normal mitochondrial function. ${ }^{18,19}$ Under stressful conditions, such as $\mathrm{Ca}^{2+}$ overload, the PTP undergoes a conformational change, it forms a non-selective channel and it allows the passive diffusion of solutes, with molecular masses up to about $1.5 \mathrm{kDa}$, but not of the proteins contained in the mitochondrial matrix. ${ }^{19}$ The osmotic force of the matrix proteins results in the accumulation of $\mathrm{H}_{2} \mathrm{O}$ and of small molecular solutes, which increased the matrix volume and might lead to the swelling of the mitochondria. An immediate consequence of the PTP opening is the collapse of the mitochondrial membrane potential $(\Delta \psi \mathrm{m})$. As a consequence, oxygen consumption is initially increased and the ATP produced by glycolysis becomes hydrolysed by the reverse operation of the $F_{0} F_{1}$ ATPase, thus leading to overall ATP depletion. Interestingly, we reported that $\mathrm{TG} 2^{-1-}$ mice show a defect in ATPase reverse activity. ${ }^{16,17}$ Under peculiar circumstances mitochondrial swelling leads to outer mitochondrial membrane rupture and further release from the intermembrane space of proapoptotic factors, such as cytochrome $\mathrm{c}$ and AIF, which triggers cell death. ${ }^{20}$

Adenine nucleotide translocator 1 (ANT1) is a bifunctional protein primarily involved in ADP/ATP exchange and it has been proposed to be among the various components of the PTP complex. ${ }^{18,20}$ Pore formation by ANT is induced by a variety of proapoptotic agents (e.g., $\mathrm{Ca}^{2+}$, atractyloside (ATRL) and Vpr HIV protein) and results to be enhanced by Bax and inhibited by $\mathrm{Bcl} 2$ and ADP. In its native form ANT is probably a dimer or an oligomer, with an even number of molecules. It has been proposed that the intermolecular cross-linking of ANT might lead to ANT dimerization, which is in favour of the channel activity of ANT. ${ }^{19}$

On the basis of these findings, we investigated whether ANT could act as a substrate for TG2.

In this study we demonstrate that ANT physically interacts with TG2 on mitochondria and acts as a substrate for TG2's activities. The physiological relevance of this phenomenon is highlighted by the fact that cells lacking TG2 show both an increased activity of the ADP/ATP transporter and an abnormal response of the translocator itself in response to stimulation, including apoptosis induction.

\section{Results}

ANT/TG2 interaction. The adenine nucleotide translocator has been recently proposed to act as a substrate for a transglutaminase-like enzymatic activity in the slime mold Physarum polycefalum. ${ }^{21}$ On the basis of this finding, we investigated whether TG2 might interact with and modify ANT1, in higher eukaryotes. To this aim, we coprecipitated both TG2 and ANT1, in protein extracts of mitochondria isolated from neuroblastoma cell lines expressing low (BE) or high (TGA) levels of TG2 protein. Figure 1a shows a representative co-IP experiment from which two relevant indications emerged: (1) ANT1 interacts with TG2 in both cell lines; (2) ANT and TG2 interaction takes place even in presence of a very low level of TG2. Note that the presence of ANT1 is clearly detectable even when TG2 is barely detectable (IP-TG2 on BE). In addition, we investigated the interaction between ANT1 and TG2 in different mice organs (Figure 1b). We performed immunoprecipitations for TG2 in protein extracts from hearts $(\mathrm{H})$ and brains $(\mathrm{B})$ of $\mathrm{TG} 2^{+/+}$ mice and revealed the presence of ANT1. It is interesting to note that, at steady state, heart's protein extracts contain more ANT1 with respect to brains' protein extracts (compare ANT signal in WCE). Interestingly, we could not coprecipitate ANT1 from brain's extracts, probably because of the low level of TG2 constitutive expression (data not shown), thus supporting the specificity of TG2/ANT1 interaction.

ANT and TG2 colocalize in mitochondria. The ANT1/TG2 interaction, revealed by the coprecipitation assays, takes place at mitochondrial level, thus TG2 must be present on mitochondria, not only at the level of their outer membrane, but also in the intermembrane space or at the inner membrane and/or matrix level. To verify this hypothesis, we performed confocal microscopy analysis (Supplementary Figure 1) as well as immunogold labelling of TG2 (Figure 1c; $5 \mathrm{~nm}$ particles), ANT1 (Figure $1 \mathrm{~d} ; 10 \mathrm{~nm}$ particles) or both proteins (Figure 1e), followed by electron microscopy visualization. We noticed that TG2 localizes both in the cytosol and at mitochondrial level, whereas ANT1 is basically localized in mitochondria. When both the proteins were labelled at the same time, there are regions in which $5 \mathrm{~nm}$ (TG2) and $10 \mathrm{~nm}$ (ANT1) gold particles were detected in close proximity, suggesting a steady-state in vivo interaction of the two proteins. It is interesting to note that TG2 seems to colocalize more with the monomeric than with the aggregate form of ANT1 (see the insets).

To get further insights on the distribution of TG2 in the various mitochondrial compartments, we treated mitochondria, isolated from TG2 overexpressing cells, with increasing concentrations of digitonin (Figure 1g; Supplementary Figure 2). The digitonin treatment leads to progressive solubilization of the outer mitochondrial membrane, allowing us to understand which of the proteins are associated/integrated in the mitochondrial membranes. ${ }^{22} \mathrm{Hsp} 60$, VDAC and ANT itself were used as internal controls for the assessment of the outer membrane solubilization and of the integrity of the mitoplasts, respectively. The obtained results suggest that the majority of the mitochondrial TG2 seem to be loosely associated with the organelle. In fact, a large percentage of 
the protein is removed by control incubation in swelling buffer (left side of the panel, digitonin $0 \%$ ) and almost all of the protein is removed at the lowest digitonin concentration $(0.1 \%)$. The right side of the panel shows the proteins remaining in the pellet, not solubilized by the digitonin treatment, and corresponding to the IMM and matrix associated proteins. It is clearly
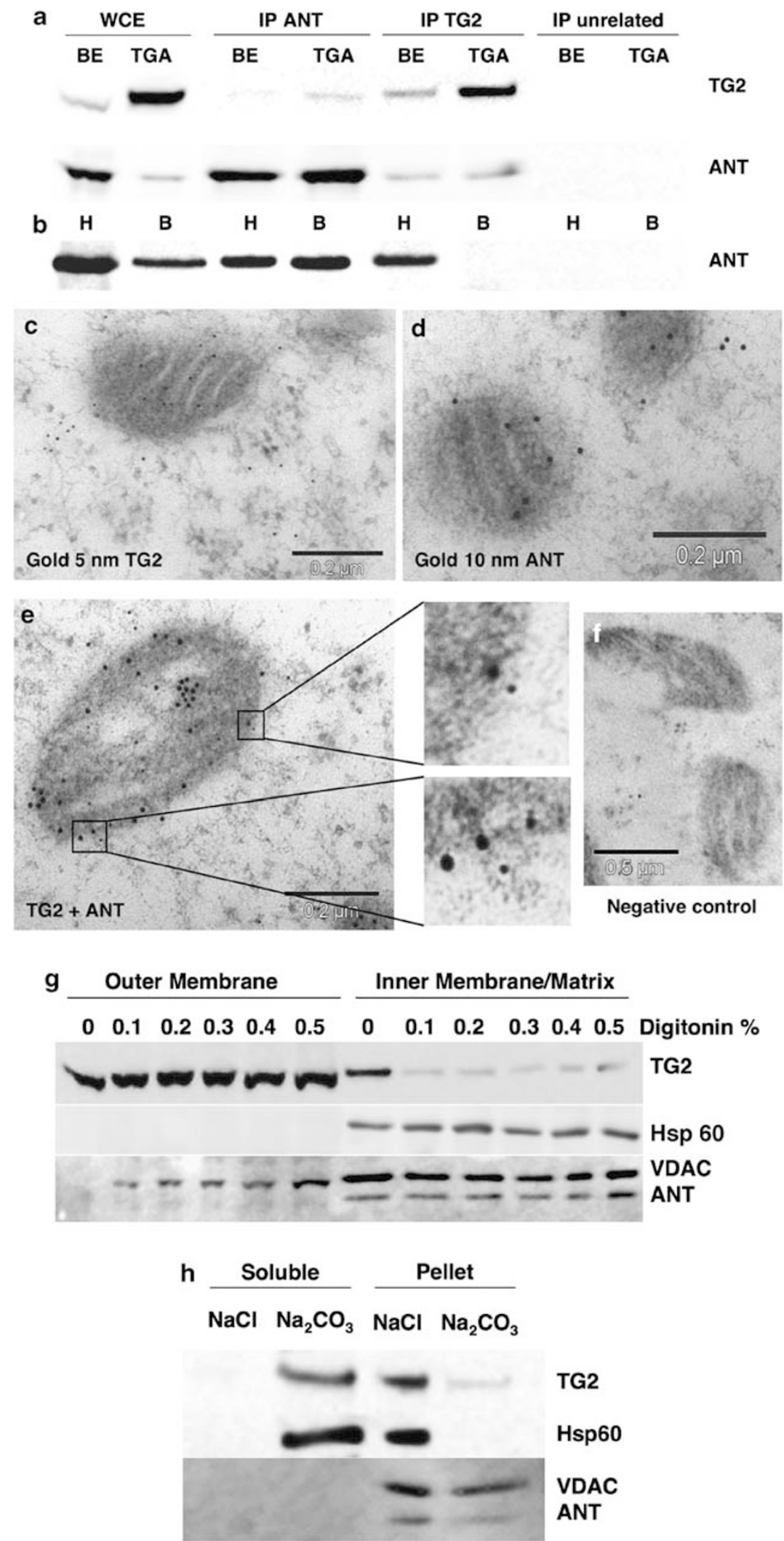
visible that a small amount of TG2 resides in strong association with the IMM or the matrix. To confirm this observation, we performed alkali extraction of isolated mitochondria (Figure 1h) and we observed that, even in this case, a small amount of TG2 is found in the pellet fraction, corresponding to the mitochondrial membranes. ${ }^{22}$ The percentage of mitochondrial TG2 that results to be associated with mitochondrial membranes and/or matrix has been estimated by measuring the relative intensity of the bands in three different digitonin treatment experiments. The mean value of TG2, normalized for the ANT1 contents, resulted to be about $5-10 \%$ of the total mitochondrial TG2. These observations supports the interaction between ANT1 and TG2, observed by the co-IPs experiments, as well as the close proximity of the two proteins revealed by electron microscopy analysis.

ANT1 acts as a TG2 substrate. We then asked the question whether ANT1 might behave as a cross-linking substrate for TG2. To this aim, we used the TG2 overexpressing cells (TGA), undergoing apoptosis in response to STS treatment, coupled to the labelling of TG2 protein substrates with 5 -(biotinamido)pentylamine (EZ-link). ${ }^{23}$ This compound enters living cells and, being cross-linked by TG2 to glutamine donor substrates, acts as a tag. ${ }^{24}$ After STS treatment, mitochondria have been purified and the biotinylated TG2 protein substrates purified by means of avidin columns. The amount of overall TG2 protein substrate on mitochondria and the presence of ANT1 among them have been detected with horseradish conjugated streptavidin and a specific anti-ANT antibody. The left panel of Figure 2a shows that few mitochondrial proteins incorporated the EZ-link substrate in untreated cells, whereas this number increases upon cell-death induction with STS. Considering that ANT has been proposed to act has a substrate for TG2 in invertebrates, we verified whether ANT1 would be present among the avidin purified TG2 protein substrates. The right panel of Figure $2 \mathrm{a}$ shows a western blot of the avidin-purified mitochondrial TG2 protein substrates probed with anti-ANT1 antibody. It is interesting to note that, in STS treated cells, the ANT1 antibody recognized not only the monomeric $(32 \mathrm{kDa})$ form of the protein but also some stable polymeric forms, ranging between 60 and $150 \mathrm{kDa}$. These polymers, which are highly resistant to denaturing conditions, become evident only after STS treatment and suggest that ANT1 becomes a
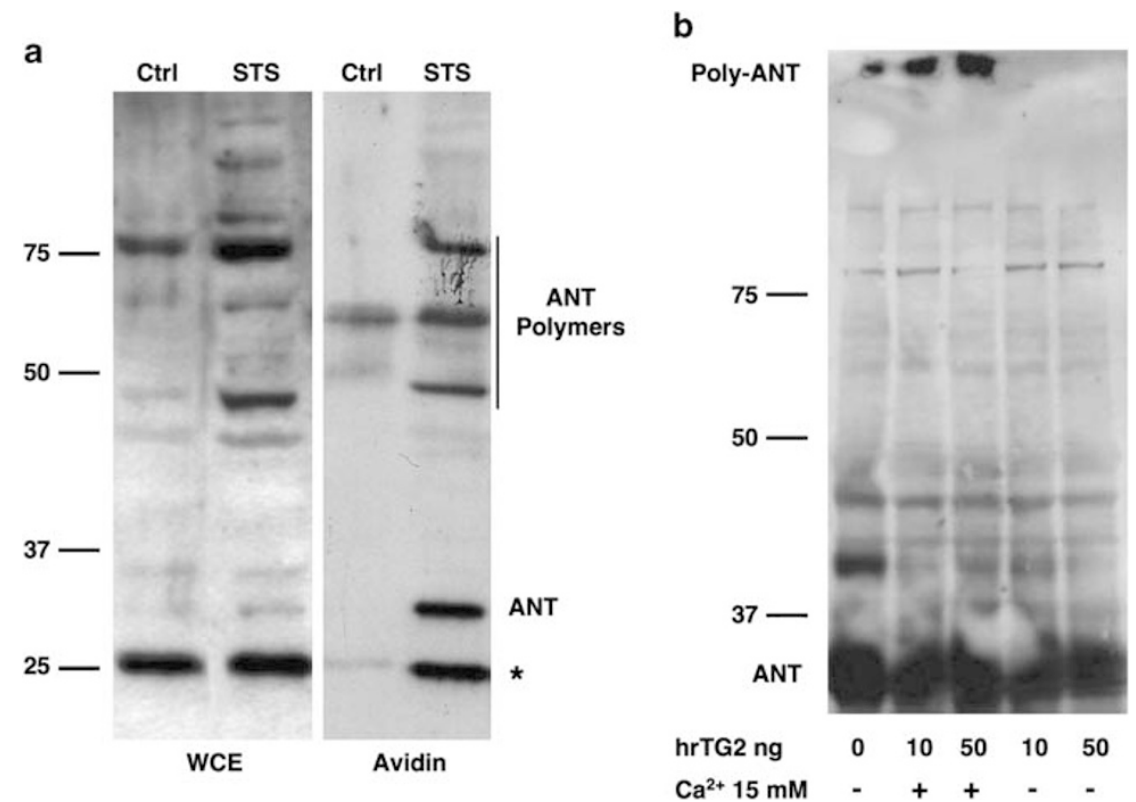

Figure 2 TG2-dependent polymerization of ANT in vivo and in vitro. (a) ANT1 is a substrate for the cross-linking activity of TG2, on cell death induction by staurosporine (STS). ANT1 is detected among the purified (avidin) TG2 protein substrates both in monomeric and polymeric forms. WCE, whole cell extracts; ${ }^{*}$, non-specific signal detected by the anti-ANT antibody. (b) In vitro TG2-dependent polymerization of ANT1. When human recombinant TG2 (hrTG2) was added to SK-n-BE(2) whole cell extracts and activated by $15 \mathrm{mM} \mathrm{Ca}^{2+}$ addition, ANT1 becomes incorporated into high molecular mass polymers, which stay at the top of the gel (Poly-ANT)

Figure 1 TG2 localizes at mitochondrial level and interacts with ANT1. (a) Coprecipitation of ANT1 and TG2 in mitochondria isolated from high (TGA) and low (BE) TG2 expressing cells. (b) Coprecipitation of ANT1 and TG2 in heart (H) and brain (B) of C57BI/6 mice. WCE, whole cell extracts; IP unrelated, unrelated antibody. (c, d, e, f) Electron microscopy analysis of ANT1 and TG2 at mitochondrial level. TG2 ( $5 \mathrm{~nm}$ gold beads) and ANT1 (10 $\mathrm{nm}$ gold beads) were detected alone (c, d) or together (e) in TG2 overexpressing cells. Insets show spots were the two proteins reside in close proximity, whereas a negative control is shown in (f). (g) Localization of TG2 in different mitochondrial compartments of TG2 overexpressing cells. Increasing concentrations of digitonin remove the outer mitochondrial membrane, as indicated by the progressive solubilization of VDAC. Hsp 60 has been used has a marker for the integrity of the internal mitochondrial membrane. (h) Alkali extraction of mitochondrial membranes. Soluble, alkali sensitive (attached or matrix proteins); Pellet, alkali resistant (inserted proteins); $\mathrm{NaCl}$, control incubation with $133 \mathrm{mM} \mathrm{NaCl}$ 
substrate for TG2 cross-linking activity only upon induction of cell death. The molecular mass of these polymers suggests that only the molecules of ANT that reside in close proximity are cross-linked by TG2's activation, although we cannot rule out the possibility that other molecules could contribute to the mass of the polymers. To further demonstrate that ANT might behave as a TG2 protein substrate, we tested the ability of human recombinant TG2 (hrTG2) to polymerize ANT1 in vitro. To this aim, we incubated whole cell extracts from cells expressing low levels of TG2 with hrTG2 in presence or absence of $\mathrm{Ca}^{2+}$, which activates the TG2 cross-linking activity. Figure $2 b$ shows a representative western blot probed with anti-ANT1 antibody of this reaction. The appearance of protein polymers of such a high molecular mass that does not enter the gel and that results to be positive to the anti-ANT1 antibody (poly-ANT at the top of the gel) is detectable only when both TG2 and $\mathrm{Ca}^{2+}$ were added to the reaction. These huge polymers completely disappeared when $\mathrm{Ca}^{2+}$ is not present, indicating that the activation of TG2 cross-linking activity is responsible for the polymerization of ANT1. ${ }^{25}$

TG2 effect on mitochondrial membrane permeabilization. We demonstrated that TG2 might interact with ANT1 under steady-state conditions and might modify it during cell-death induction. On the basis of these observations and on the role exerted by ANT1 on mitochondria, we investigated whether the presence or the absence of TG2 might affect the mitochondrial membrane potential and its permeabilization. To this aim, we generated mouse embryonic fibroblasts (MEFs) from $\mathrm{TG} 2^{+1+}$ and $\mathrm{TG} 2^{-1-}$ mice. We measured the mitochondrial membrane potential in intact cells by using the specific probes $5-5^{\prime}, 6-6^{\prime}$-tetrachloro$1,1^{\prime}, 3,3^{\prime}$-tetraethylbenzimidazol-carbocyanine iodide (JC-1) or tetramethylrhodamine ester (TMRM) in the presence of three different 'mitochondriotropic' agents: (1) cyclosporine A (CyA), which prevents permeability transition via the inhibition of cyclophilin D binding to ANT; (2) bongkrekic acid (BA), a transport inhibitor that blocks the ANT porter by binding to the inward-facing conformation; (3) ATRL, another transport inhibitor able to block the ANT porter by binding to the outward-facing conformation (in contrast to BA). ${ }^{19}$ As a control for the depolarization of the mitochondrial membrane we used a low dose of carbonyl cyanide p-(trifluoromethoxy) phenylhydrazone (FCCP; Figure 3a, fifth panel). Quantitative flow cytometry analysis, performed by using JC-1 metachromatic probe (Figure $3 a$, dot plots from a representative experiment are shown), revealed the presence of a significantly higher percentage of cells with hyperpolarized mitochondria (see boxed areas) in TG2 ${ }^{+/+}$(48 $\pm 5 \%$; first row, first panel) with respect to $\mathrm{TG}^{-1-}$ MEFs $(24 \pm 4 \%$; second row, first panel), independently from any treatment. In addition, either CyA (second panel) or BA (third panel) was able to significantly reduce the percentage of cells with hyperpolarized mitochondria in $\mathrm{TG} 2^{+/+} \mathrm{MEFs}$ only (first row).

Strikingly, a very different effect was induced by ATRL in the two cell lines. In fact, in TG2 ${ }^{+/+}$MEFs (first row, fourth panel) the effect of ATRL was very similar to that observed for CyA and BA (i.e., a reduction of mitochondrial membrane hyperpolarization). By contrast, in $\mathrm{TG} 2^{-1-} \mathrm{MEFS}, \mathrm{ATRL}$ treatment induced a strong depolarization of mitochondrial membrane in about $30 \%$ of the cells (second row, fourth panel, see numbers under the dashed line). Figure $3 \mathrm{~b}$ shows a summary of the results obtained by using TMRM as an alternative probe. Data are reported as median fluorescence values from three independent experiments. In addition, similar results were obtained by treating with ATRL cells that express low (SK-N-BE(2)) or high (TGA) levels of TG2 protein (see Supplementary Figure 3).

We then verified whether the dramatically different effect induced by ATRL in these cell lines might be due to their own constitutive mitochondrial features. To this aim, we investigated the ability of ATRL to evoke swelling in mitochondria isolated from either $\mathrm{TG} 2^{+/+}$or TG2 $2^{-/-}$MEFs. The purity degree of our mitochondrial preparation was checked by flow cytometry to exclude the presence of remnants of other intracellular organelles, such as vesicles from endolysosomal compartment and/or Golgi apparatus. Results obtained (Figure 3c) show that the isolated mitochondria were negative either for the endolysosomal compartment antigen Rab5 or for GM130, a protein that characterizes Golgi apparatus, although were strongly positive for the mitochondrial protein VDAC-1 (voltage-dependent anion channel). We found that, as for intact cells, isolated mitochondria derived from $\mathrm{TG} 2^{+1+}$ MEFs were characterized by a higher MMP, with respect to those isolated from TG2 $2^{-1-}$ cells (Figure $3 d$ ). In fact, the median fluorescence intensity after TMRM staining was $49.5 \pm 6.1$ for TG2 ${ }^{+/+}$(black line) and $27.3 \pm 4.9$ for TG2 $2^{-/-}$ (grey line). The upper panels of Figure $3 e$ show a representa-

Figure 3 Mitochondria of TG2 ${ }^{+1+}$ and TG2 ${ }^{-1-}$ MEFs display impaired membrane polarization and sensitivity to stimuli. (a) Biparametric flow cytometry analysis of MMP after staining with JC-1 in TG2 ${ }^{+1+}$ and TG2 ${ }^{-1-}$ cells untreated (Ctrl) or treated for $48 \mathrm{~h}$ with cyclosporine A (CyA), bongkrekic acid (BA) or atractyloside (ATRL). Negative controls were performed by treating cells with FCCP. Numbers reported in the boxed area represent the percentages of cells with hyperpolarized mitochondria. In the area under the dashed line, the percentage of cells with depolarized mitochondria is reported. Numbers represent the mean \pm S.D. of the median fluorescence values among four independent experiments. Dot plots from a representative experiment are shown. (b) Monoparametric flow cytometry analysis of MMP after cell staining with TMRM. Results are reported as mean \pm S.D. of the median fluorescence intensity among three independent measurements. $\left(^{*}\right) P<0.01$ versus Ctrl; $\left({ }^{\circ}\right) P<0.01$ versus $\left(^{\star}\right)$. (c) Flow cytometry analysis of mitochondria preparation after staining with MAb to Rab5 (deep grey), GM130 (light grey) or VDAC-1 (black full), to test its degree of purity. The negative control (IgG1 + anti mouse-Alexa 488) is represented by the dashed line. Numbers represent the median values of fluorescence intensity. (d) Membrane potential of mitochondria isolated from TG2 ${ }^{+1+}$ (grey line) or TG2 ${ }^{-1-}$ (black line) MEFs after staining with TMRM. Numbers represent the median values of fluorescence intensity. (e) Effect of ATRL on isolated mitochondria. The swelling profile of the mitochondria isolated from TG2 ${ }^{+1+}$ (left panels) or TG2 ${ }^{-1-}$ (right panels) as monitored by means of variations in TMRM fluorescence, as a function of time. Numbers in grey areas of each plot represent the percentage of mitochondria that underwent MMP decrease. Results obtained in a representative experiment are shown. Note that: (i) calcium $300 \mu \mathrm{M}$, used as positive control, induce a similar and rapid loss of mitochondrial membrane potential in both cell lines, whereas calcium $10 \mu \mathrm{M}$ was ineffective; (ii) ATRL was able to induce mitochondrial swelling in mitochondria isolated from TG2 ${ }^{-1-}$ MEFs only 
tive profile of the mitochondrial swelling induced by $\mathrm{Ca}^{2+}$ in $\mathrm{TG}^{+/+}$(left dot plots) and TG2 ${ }^{-1-}$ (right dot plots) purified mitochondria, monitored by means of the variations in TMRM fluorescence as a function of time. The addition of $10 \mu \mathrm{M}$ $\mathrm{Ca}^{2+}$ did not induce per se any sign of mitochondrial swelling, whereas $300 \mu \mathrm{M} \mathrm{Ca}^{2+}$ induced swelling in a significant a
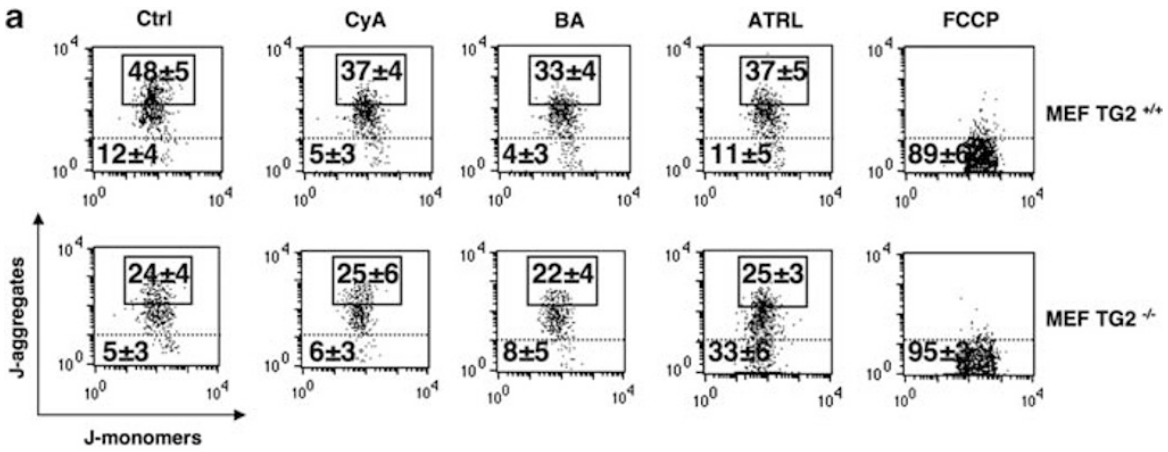
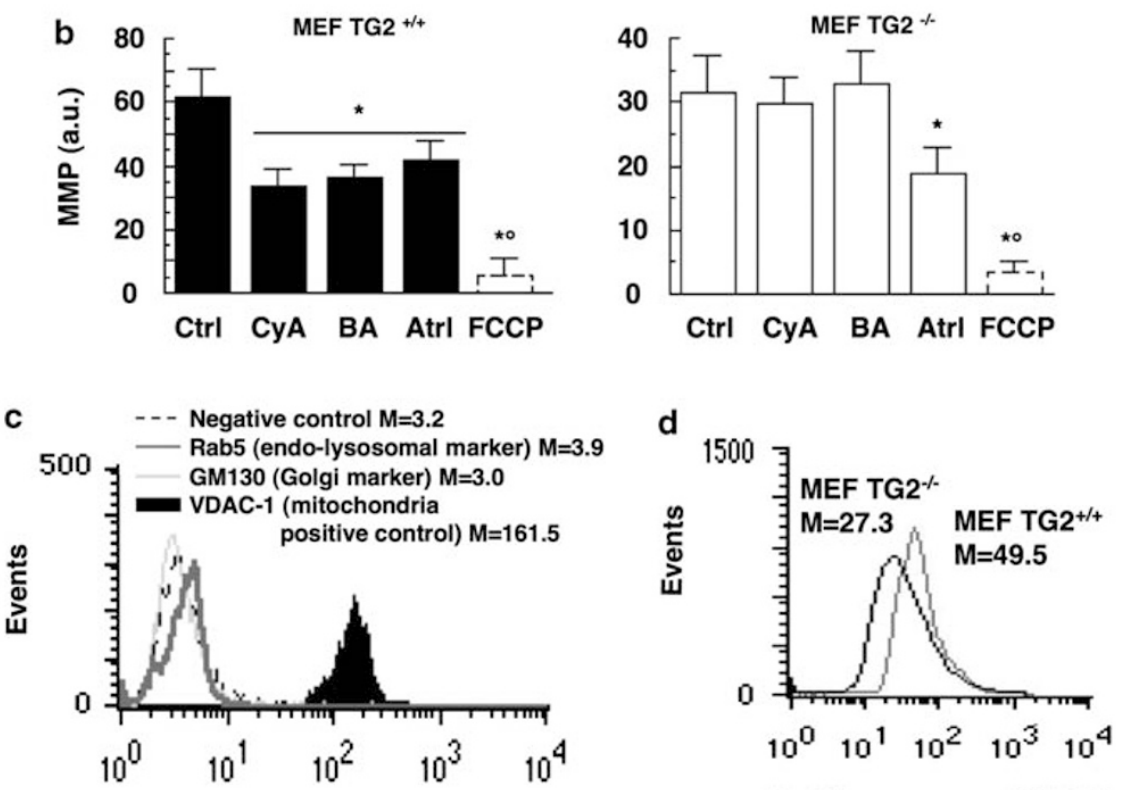

Green fluorescence

Red fluorescence (TMRM)
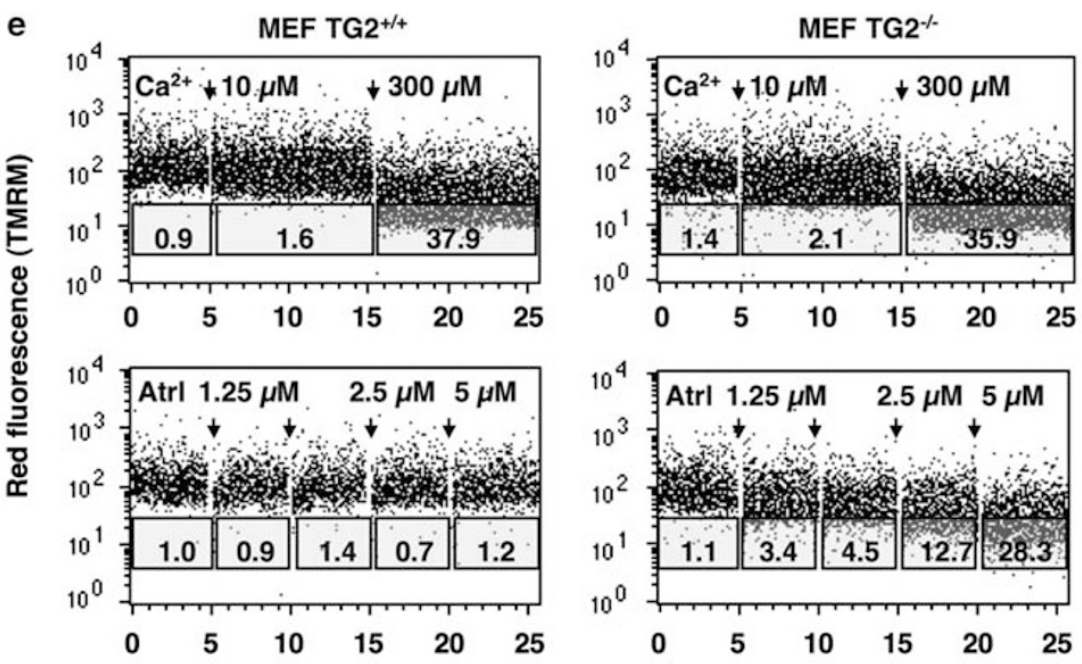

Time (minutes) 
percentage of mitochondria either in TG2 ${ }^{+1+}(37.9 \pm 7.3)$ or in $T G 2^{-l-}(35.9 \pm 6.4)$ MEFs. It is interesting to note that the effects of $A T R L$ were evident only in mitochondria purified from $\mathrm{TG}^{-1-}$ MEFs (bottom right dot plot), where it induced a dose-dependent mitochondrial membrane depolarization (starting from $1.25 \mu \mathrm{M}$ up to $5 \mu \mathrm{M}$ ). By contrast, ATRL was unable to induce any sign of mitochondrial swelling in TG2 ${ }^{+/+}$ MEFs purified mitochondria, either per se (bottom left dot plot) or after $10 \mu \mathrm{M} \mathrm{Ca}{ }^{2+}$ addition (not shown). Low $\mathrm{Ca}^{2+}$ concentration, although not generally mandatory for mitochondrial swelling, has been reported to be indispensable for mitochondrial response evoked by some mitochondrial perturbating agents. ${ }^{26}$ These data suggested that TG2 might exert a constitutive regulation of ANT1, which might be associated with a structural change in the protein itself, as highlighted by the differences observed in the steady-state MMP as well as by the different sensitivity to ATRL of TG2 $2^{-/-}$ MEFs with respect to the $\mathrm{TG} 2^{+/+}$ones.

TG2/PDI activity regulates the oligomerization of ANT1. The data obtained by labelling mitochondrial TG2's protein substrates (Figure $2 \mathrm{a}$, left panel) suggested the possibility that the steady-state regulation of the ADP/ATP transporter is not dependent on the cross-linking activity of the enzyme, because no incorporation of EZ-link was observed before cell-death induction. In addition, when we carried out western blot analysis of $\mathrm{TG} 2^{+/+}$and $\mathrm{TG} 2^{-l-}$ protein extracts under reducing conditions, we observed only one major band corresponding to the molecular mass of the monomeric form of ANT1 (Figure 4; + DTT). These results support the hypothesis that, under physiological conditions,

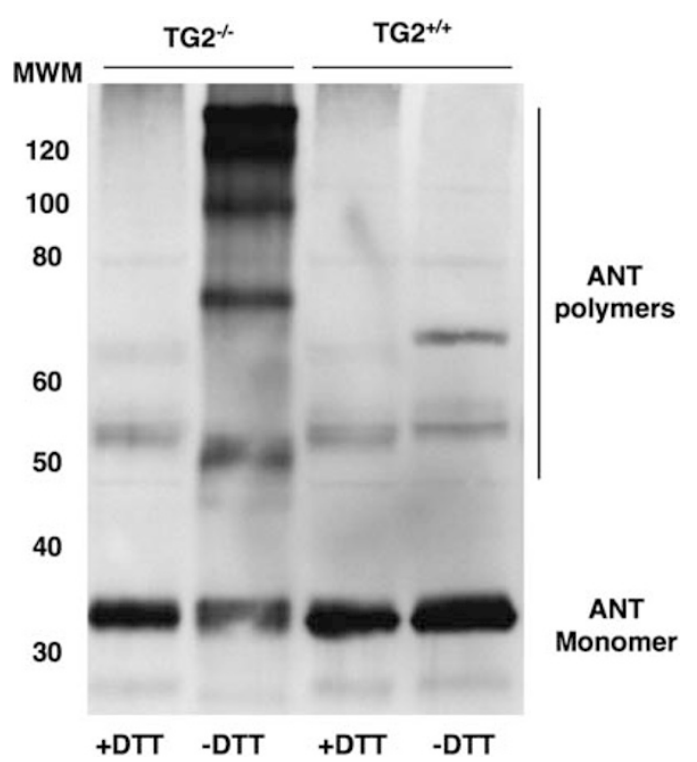

Figure 4 ANT1 is substrate for TG2-PDI activity in the heart. Protein extracts from isolated hearts mitochondria of $\mathrm{TG} 2^{+1+}$ or $\mathrm{TG} 2^{-1-}$ mice where separated under reducing (+ DTT) or non-reducing (-DTT) conditions and probed with antiANT antibody. The presence of ANT1 polymers, under non-reducing conditions, is detectable in a range spanning between 50 and $200 \mathrm{kDa}$ only in protein extracts of mitochondria isolated from $\mathrm{TG}^{-1-}$ mice the TG2 cross-linking activity does not contribute to the posttranslational modification of ANT1. However, considering that TG2 is a multifunctional enzyme and not exquisitely a cross-linking one, we asked the question whether an alternative TG2-dependent molecular mechanisms might explain our findings. It has been reported that TG2 might function as a PDI and it has been shown recently that, under physiological conditions, TG2 is able to catalyse the formation of disulphide bonds of other mitochondrial proteins. ${ }^{7,16}$ To assess whether the TG2-dependent formation of disulphide bonds is involved in the homoeostasis of ANT1 at mitochondrial level, we performed western blot, under reducing and non-reducing conditions, of protein extracts from mitochondria isolated from $\mathrm{TG}^{+/+}$and $\mathrm{TG} 2^{-1-}$ mice hearts. Under reducing conditions, the levels of the monomeric form of the protein are comparable in both $\mathrm{TG} 2^{+1+}$ and $\mathrm{TG} 2^{-l-}$ mice and no other bands were observed (Figure 4; +DTT). By contrast, when disulphide bonds are preserved, under non-reducing conditions, we detected the presence of high molecular mass ANT1 protein polymers in mitochondria isolated from $\mathrm{TG}^{-1-}$ mice hearts. It is interesting to note that those polymers were not observed in the mitochondrial fractions obtained from $\mathrm{TG}^{+/+}$mice (Figure 4; -DTT). These results indicate the existence of an inverse correlation between the formation ADP/ATP transporter polymers and the presence of TG2 in vivo. To confirm that TG2 is responsible of the observed effects, we made an in vitro reconstitution experiment by adding human recombinant TG2 to $\mathrm{TG} 2^{-1-}$ mitochondria protein extracts. We observed that TG2 incubation leads to a marked reduction of the ANT polymers, restoring, at least partially, the pattern of ANT polymers observed in the $\mathrm{TG}^{+1+}$ mice (see Supplementary Figure 4).

To verify whether the increased oligomerization of ANT1 observed in $\mathrm{TG}^{-1-}$ mice was reflected in a modification of its transporter activity, we measured the ADP/ATP exchange in mouse tissues obtained from both $\mathrm{TG}^{+/+}$and $\mathrm{TG} 2^{-/-}$mice. The exchange activity of the ANT translocator resulted to be increased in the mitochondria of $\mathrm{TG}^{-1-}$ mice, namely of about twofold in the heart and half-fold in the brain, whereas no differences have been detected in mitochondria isolated from the liver (see Supplementary Figure 5). These results clearly indicate that in vivo the lack of TG2 leads to a marked increase of the transporter's activity in the heart, and to a lesser extent in the brain and in the liver, and they correlate with the presence of ANT1 polymers in the mitochondria of $\mathrm{TG}^{-1-}$ mice.

TG2 effects on apoptosis induction. The susceptibility to and the execution of apoptosis strongly rely on both mitochondrial membrane permeabilization and ANT1. ${ }^{20}$ For this reason we analysed the responsiveness of $\mathrm{TG} 2^{+/+}$and $\mathrm{TG}^{-1-}$ MEFs to the glucose analogue and glycolysis inhibitor deoxy-D-glucose (DDG) as well as the possible modulation of cell death exerted by the mitochondriotropic agents we used previously. Annexin V-FITC/PI staining and flow cytometry analysis of cell death (Figure 5) clearly indicated that: (1) in absence of any stimulus, TG2 ${ }^{-1-}$ MEFs displayed lower levels of 'spontaneous' apoptosis, in comparison with the $\mathrm{TG}^{+/+}$counterpart; (2) DDG-treatment 

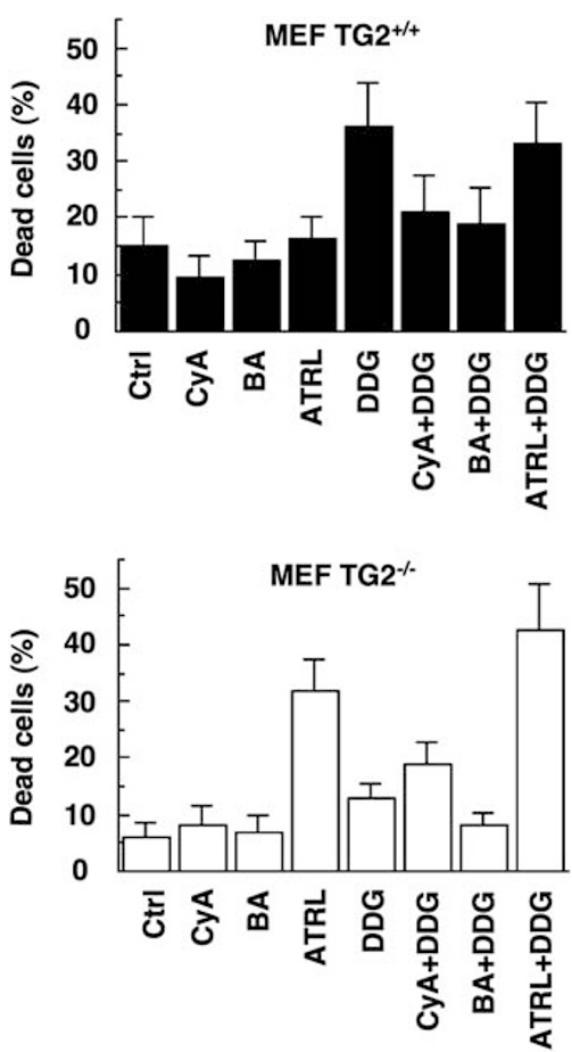

Figure 5 Differential sensitivity to apoptosis induction of $\mathrm{TG}^{+1+}$ versus $\mathrm{TG}^{-1-}$ MEFs. Apoptosis quantification after double staining with Annexin V-FITC/ presidium iodide in TG2 ${ }^{+/+}$(black bars) and TG2 ${ }^{-1-}$ (white bars) MEFs. Results are reported as mean \pm S.D. among five independent experiments

was an efficient apoptotic inducer in TG2 ${ }^{+/+}$MEFs but not in $\mathrm{TG}^{-/-}$ones. In fact, although we could observe about $40 \%$ of DDG-induced apoptosis in TG2 $2^{+/+}$MEFs, the percentage of apoptotic cells was significantly lower (about 15\%) in $\mathrm{TG}^{-1-}$ ones. It is interesting to note that both CyA and BA were able to significantly prevent DDG-induced apoptosis in $\mathrm{TG}^{+1+}$ MEFs, whereas ATRL was not. On the other hand, in $\mathrm{TG}_{2}{ }^{-1-}$ MEFs neither CyA nor BA had an effect on cell death, whereas ATRL showed a drastic and specific effect.

It has been widely reported that, during apoptosis, some proteins (e.g., Bax and $\mathrm{Bcl}-2$ ) might relocalize to mitochondria, interact with ANT1 and regulate the dissipation of the mitochondrial transmembrane potential. ${ }^{27}$ On the basis of the results obtained so far, we decided to analyse the localization of Bax and ANT1 in TG2 ${ }^{+/+}$and TG2 ${ }^{-/-}$MEFs, by means of intensified video microscopy (Figure 6a). As expected, we found no colocalization of these two proteins in untreated control cells both in $\mathrm{TG} 2^{+/+}$and $\mathrm{TG} 2^{-/-}$MEFs. By contrast, after DDG treatment, we observed the recruitment of Bax to the mitochondria and its colocalization with ANT1 in TG2 ${ }^{+/+}$ but not in $\mathrm{TG} 2^{-1-}$ MEFs. We then investigated the localization of Bax and ANT1 after treatment with STS, which was able to induce the same extent of cell death in the two cell lines (data not shown). Interestingly, as detected for DDG, STS also induces ANT1/Bax colocalization in TG2 ${ }^{+/+}$but not in $\mathrm{TG}^{-/-}$MEFs. These observations were supported by morphometric analysis, performed on at least 100 cells/ sample. The data obtained (Figure $6 \mathrm{~b}$ ) clearly indicated that the ANT1/Bax colocalization, negligible in untreated cells, was significantly increased both in DDG- and STS-treated $\mathrm{TG} 2^{+/+}$MEFs (black bars). By contrast, we failed to reveal ANT1/Bax association either in DDG- or STS-treated TG2 ${ }^{-1-}$ MEFs (white bars). To get further insights on this ANT1/Bax interaction, we used flow cytometry fluorescence resonance energy transfer (FRET) methodology ${ }^{28}$ on isolated mitochondria. The sensitivity of this approach allowed us to point out that, at mitochondrial level, the molecular association of ANT1 with Bax was detectable only in TG2 ${ }^{+/+}$MEFs and only after apoptotic stimulation, either by STS or by DDG (Figure 6c, upper panels). By contrast, in TG2 ${ }^{-1-}$ MEFs the ANT1/Bax association was negligible either in untreated or in STS or DDG-treated cells (Figure 6c, lower panels). These observations were quantified by calculating the FRET efficiency (Figure 6d), as described in the Materials and methods section. This quantitative analysis indicated a significant FE only in STS or DDG treated TG2 ${ }^{+1+}$ MEFs but not in the $\mathrm{TG} 2^{-1-}$ ones. As a final assay we made coprecipitation assays for ANT1 on mitochondria isolated from $\mathrm{TG}^{+/+}$and $\mathrm{TG}^{-1-}$ MEFs, treated or not with STS and revealed the presence of Bax (Figure 6e). As expected, ANT1/Bax interaction was detected only in TG2 ${ }^{+/+}$MEFs and not in $\mathrm{TG}^{-1-}$ ones, both in untreated and STS-treated cells. These results confirmed the data obtained with fluorescence microscopy and FRET analysis and suggest that the presence of TG2 is mandatory to enable and/or stabilize the association of ANT1 with Bax.

\section{Discussion}

The adenine nucleotide translocator is one of the most abundant mitochondrial protein and it has been proposed to be one of the components of the permeability transition pore complex (PTPC), ${ }^{20}$ whose exact structure has not yet been completely clarified. In addition, the role of ANT as an essential structural component or as a modulator of PTPC, able to regulate the pore opening depending on the cellular physiology, is currently being intensively debated (for details, see references ${ }^{35,36}$ ).

The major physiological role of ANT is to rapidly transport ATP out of the mitochondria and ADP back into it. ${ }^{37}$ Four isoforms of ANT, each of about 300 amino acids in length (33 kDa) and sharing about $90 \%$ of sequence identity, have been identified in humans. ANT1 results to be primarily expressed in highly differentiated tissues, such as brain, heart and skeletal muscle. ${ }^{38}$ ANT2 is expressed only in rapidly proliferating cells, such as myoblasts and tumours, but not in normal adult tissue. ${ }^{19}$ ANT3 is ubiquitously expressed in all tissues and ANT4 occurs mainly in liver, testis and undifferentiated embryonic stem cells. ${ }^{39}$

ANT1 is localized in the IMM and it might interact with VDAC and cyclophilin D (CyP-D), at the mitochondrial membranes contact sites. Two distinct conformations of ANT1 have been defined. The ' $c$ ' conformation, in which the non-membrane permeable inhibitor carboxyatractyloside (ATRL) binds to ANT from the cytosolic surface, and the ' $m$ ' conformation, in 


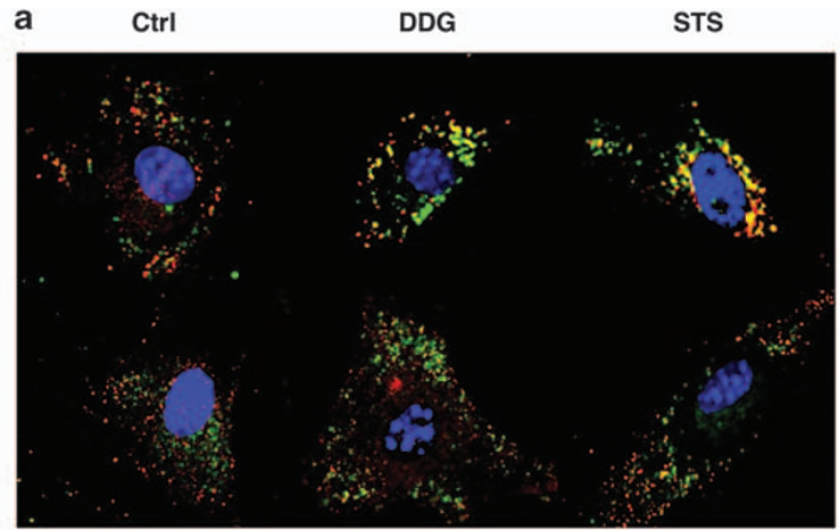

TG2 $^{+/+}$

TG2 $^{-1-}$
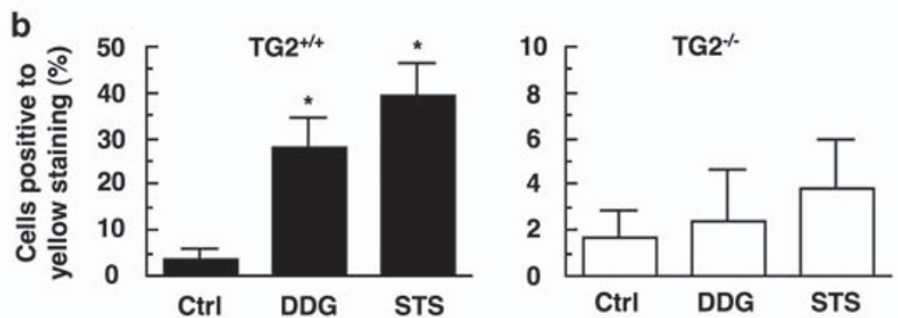

C
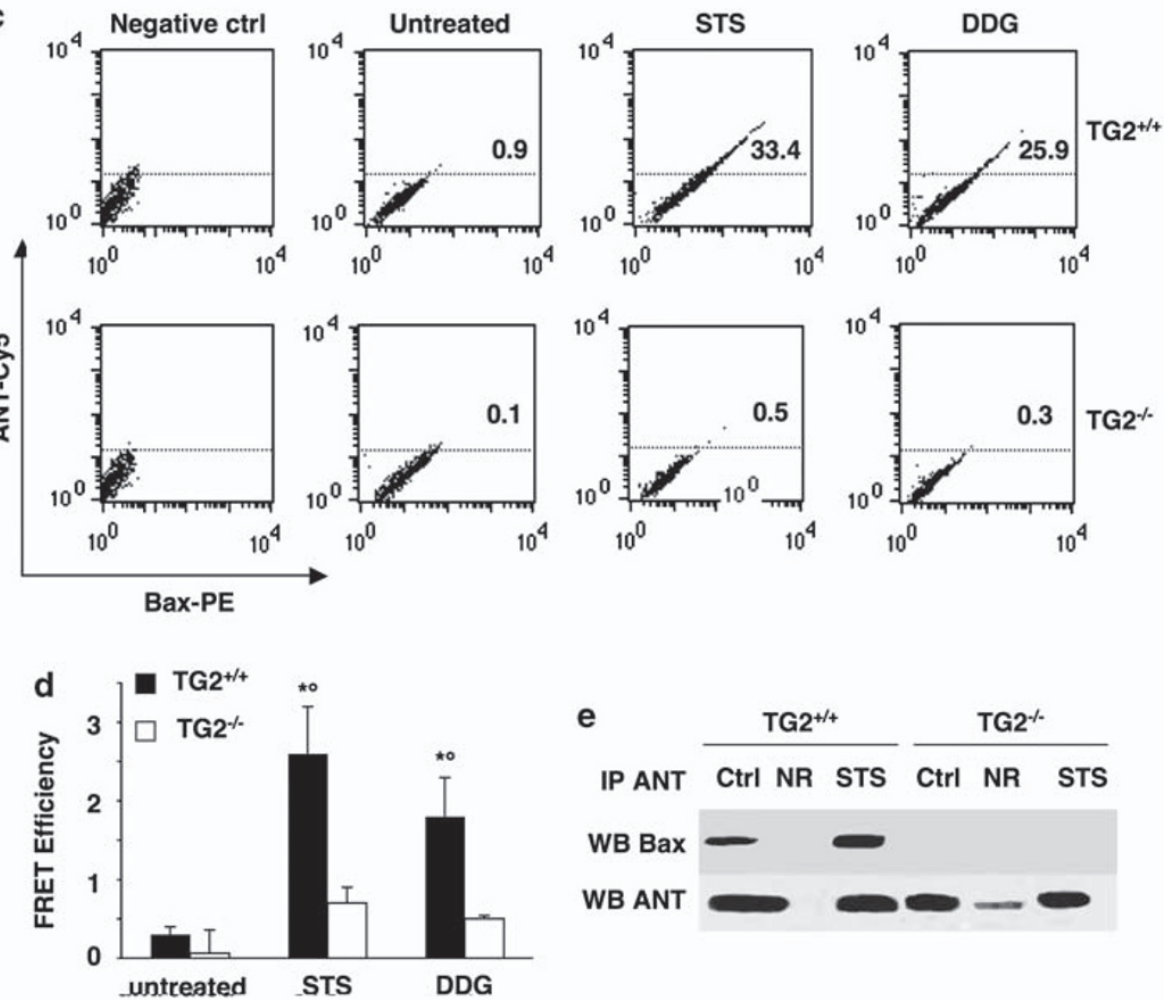

e
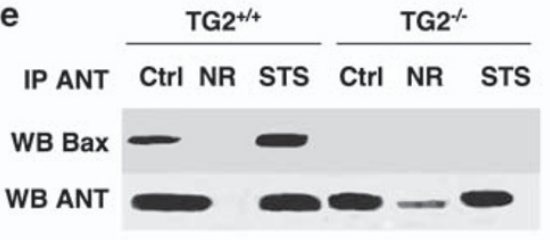

Figure 6 BAX/ANT interaction is dependent on the presence of TG2. (a) IVM analysis of ANT and Bax colocalization in TG2 ${ }^{+1+}$ and TG2 $2^{-1-}$ MEFs after treatment with the glycolysis inhibitor deoxy-D-glucose (DDG) and staurosporine (STS). The colocalization of the two proteins, clearly visible in TG2 ${ }^{+1+} \mathrm{MEFs}$, is barely detectable in $\mathrm{TG}^{-l-}$ ones. (b) Quantitative evaluation of the ANT1/Bax interaction, performed by analysing at least 100 cells/sample. Results are reported as percentage of cells showing yellow staining as a consequence of ANT1 (green) and Bax (red) colocalization (see Supplementary Figure 6). (c). Flow cytometry analysis of ANT1/Bax association in isolated mitochondria by FRET technique. FRET from acceptor to donor, in terms of percentage of FL3 positive events, in TG2 ${ }^{+1+}$ MEFs (first row) and TG2 ${ }^{-l-}$ MEFs (second row). Numbers represent the percentage of mitochondria in which ANT1/Bax association occurred. Results obtained in one representative experiment out of three are shown. (d) Evaluation of FE according to the Riemann's algorithm. Results represent the mean \pm S.D. from three independent experiments. ( $\left.{ }^{*}\right)$ Indicates $P<0.01$ versus untreated TG2 ${ }^{+1+}$ MEFs; $\left({ }^{\circ}\right)$ indicates $P<0.01$ versus the corresponding treatment of TG2 ${ }^{-1-}$ MEFs. (e). ANT1/Bax interaction assessed by immunoprecipitation of ANT1. ANT1 was precipitated from mitochondria belonging to untreated (Ctrl) or staurosporine (STS) treated TG2 ${ }^{+1+}$ and TG2 ${ }^{-1-}$ MEFs. NR: not related antibody 
which the membrane permeable inhibitor BA binds to the matrix surface of ANT. ${ }^{19}$

TG2 catalyses a $\mathrm{Ca}^{2+}$-dependent cross-linking reaction that leads to the polymerization of various protein substrates. The drop of intracellular GTP concentration and the increase of intracellular $\mathrm{Ca}^{2+}$ levels, observed during apoptosis induction, are responsible for the activation of TG2 crosslinking activity, which results in the assembly of highly crosslinked intracellular protein polymers. In addition, TG2 might also behave as $\mathrm{BH} 3-$ only protein and it might act as an upstream regulator of the mitochondrial pathway of apoptosis. ${ }^{32}$ TG2 localization on mitochondria causes a steady-state hyperpolarization of mitochondrial membrane and a rapid loss of MMP and release of cytochrome c, upon induction of apoptosis. ${ }^{40}$ To understand by which mechanism TG2 can alter mitochondrial physiology, we investigated which of the mitochondrial protein(s) might act as substrates for the various activities of TG2.

We have previously observed that the TG2 BH3-like domain allows the enzyme to localize at mitochondrial level where it interacts with and polymerizes Bax and Bak, after cell-death induction. ${ }^{32}$ In addition, we demonstrated that mitochondrial localized TG2 exerts a PDI activity and, under physiological conditions, it contributes to the correct assembly of some subunits of the mitochondrial respiratory complexes. Lack of these TG2-dependent post-translational modifications impairs the activity and the homoeostasis of the respiratory chain, resulting in the alteration of the ATP synthesis levels. ${ }^{16}$

We show here that TG2 is involved in the mitochondrial pathway of cell death not only via the interaction with Bax and Bak and their $\mathrm{Ca}^{2+}$-dependent polymerization, but also by interacting with and modifying the activity of ANT1. It is interesting to note that the recruitment of Bax to mitochondria is significantly affected, in the absence of TG2, very likely explaining the reduced ability of $\mathrm{TG}^{-/-} \mathrm{MEFs}$ to undergo apoptosis. By contrast, TG2 ${ }^{-/-}$MEFs are exquisitely sensitive to the action of ATRL, suggesting that, in the absence of TG2, ANT1 is not able to acquire an appropriate conformation and it becomes particularly sensitive to this agent. Furthermore, we cannot rule out the possibility that a more complex remodelling of mitochondria, and in particular of the mitochondrial targets of the mitochondriotropic agents cyclosporine A, bongkrekic acid and ATRL, could occur in absence of TG2. We might hypothesize that ATRL acts 'upstream', in comparison with the other agents, thus resulting in a sequential cascade of events that should still be assessed.

We demonstrate here that TG2 localizes in mitochondria, under physiological conditions, and its presence could be revealed as associated to the outer membrane and/or in the intermembrane space as well as integral to the inner membrane and/or in the mitochondrial matrix. This localization allows the protein to regulate the activity of the ATP/ADP transporter, as indicated by its constitutive higher activity observed in the heart, and to a lesser extent in the brain, of $\mathrm{TG}^{-1-}$ mice. Maintenance, or even acceleration, of ANT1 transporter activity exerts protective effects on impaired heart tissue, by stabilizing cellular energy metabolism, preventing cell death and consequently improving myocardial contractility. ${ }^{18}$
Genetic inactivation of the nuclear-encoded mitochondrial ANT1 (Ant1 $1^{-/-}$mice), results in lactic acidosis and mitochondrial cardiomyopathy and myopathy, the latter involving hyperproliferation of mitochondria, induction of oxidative phosphorylation (OXPHOS) enzymes, increased reactive oxygen species (ROS) and excessive mtDNA damage. ${ }^{13,18}$ Genetically or physiologically restricted ANT1 functionality associates with insufficient energy supply and the resulting induction of apoptosis leads to severe cardiac disturbance. $^{18,37}$ In contrast, to counteract myocardial stress, heart tissue developed cell protecting gene programs, including ANT1 upregulation, to stabilize energy supply and to suppress the apoptotic process. ${ }^{18}$ It is interesting to note that $\mathrm{TG} 2^{-1-}$ mice are particularly sensitive to ischemia/reperfusion, as a consequence of their impairment of ATP homoeostasis. ${ }^{15}$ Furthermore, recent findings support an important role for ANT1 defects in the development of Type II diabetes, which has been associated with an increased ROS production. ${ }^{13}$

We show here that TG2's protein PDI activity is physiologically involved in the maintenance of the mitochondrial physiology, by modulating the level of ANT1 polymers in the heart. PDIs are unique and multifunctional enzymes that might function as disulphide oxidases, reductases or isomerases as well as chaperones. We show that the absence of TG2 leads to ANT1 polymerization, through the formation of dithiol cross-linking, which is not detected in the presence of TG2. On this basis, we might hypothesize that TG2 could regulate the formation of ANT1 dithiols, by acting as a PDI, thus reducing the level of the pool of polymerized protein and then reducing its transporter activity. This regulation can also be affected by the physical interaction between TG2 and ANT1 that can also regulate the ADP/ATP transporter function by sequestering ANT1 and thus preventing its oligomerization (see the proposed model in Figure 7).

In conclusion, our data suggest a complex role exerted by TG2 on mitochondrial physiology. Under physiological conditions, the PDI activity of the enzyme might contribute to the stabilization of the respiratory complex I as well as of the monomeric/polymeric status ANT1, and consequently to the overall ATP homoeostasis (Table 1). In fact, TG2 ${ }^{-1-}$ mice show an impairment of ATP production that is partially buffered by the increased activity of both respiratory

TG2 regulation of the ADP/ATP transporter activity

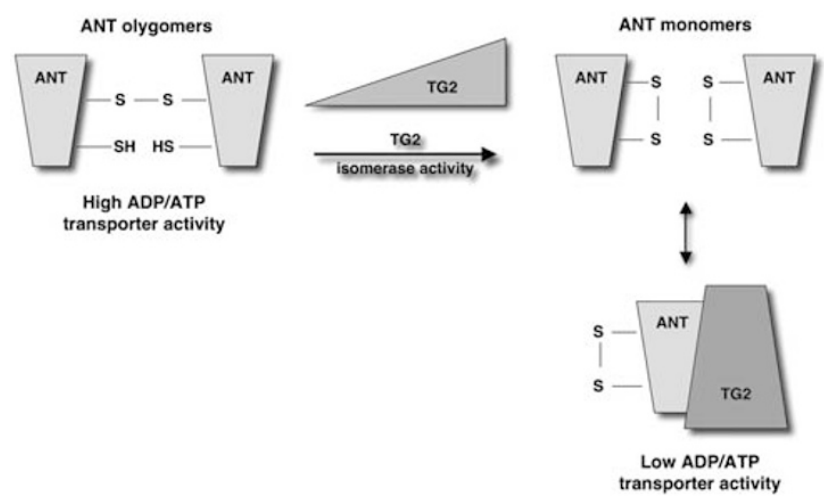

Figure 7 Proposed model for the action exerted by TG2 on ANT. 
Table 1 Summary of the mitochondrial alteration observed in $\mathrm{TG}^{-/-}$mice

\begin{tabular}{lll}
\hline & TG2 $^{-1-}$ mice & Reference \\
\hline Respiratory complex I & $50 \%$ Reduction & 16,17 \\
Respiratory complex II & $30 \%$ Increase & 16,17 \\
ATP Production & $40 \%$ Reduction (heart) & 16 \\
ATP Synthase reverse & Impaired & 15 \\
activity & & \\
ATP/ADP carrier activity & Increased (heart) & This paper \\
Mitochondrial & Increased & 40 \\
membrane potential & & \\
Bax recruitment & Impaired & This paper \\
Apoptosis & Reduced & This paper and \\
& & reference \\
\end{tabular}

complex II and ANT1 (Table 1). In keeping with these findings, heart-specific transgenic rats overexpressing $\mathrm{ANT} 1^{41}$ and leading to enhanced mitochondrial ANT functionality, show an increase in the activity of respiratory complexes II and IV, indicating a tight energetic coupling. By contrast, under stressful conditions, TG2 contribute to the recruitment of Bax on the mitochondria and the $\mathrm{Ca}^{2+}$-dependent activation of its cross-linking activity leads to the polymerization of ANT1, thus contributing to the irreversible damage of the mitochondria. A very recent paper, ${ }^{42}$ reported the obligate contribution of ANT in the execution of developmental and homoeostatic cell death in Caenorhabditis elegans. The physical and functional interaction between CED-9, the sole multi-domain Bcl-2 protein of $C$. elegans, and ANT emphasizes the existence of an intricate, phylogenetically conserved crosstalk between Bcl-2 family proteins and constituents of the PTPC, in particular the ANT orthologue WAN-1. The results we reported here might contribute to shed additional light on the role played by ANT and TG2 in the regulation of life/death decision(s) that takes place at mitochondrial level, in the physiological regulation of the ATP/ADP exchange as well as in the recruitment of Bax to the ANT1 complex during the early stages of cell death, preceding the opening of the PTPC.

In conclusion, our findings open new avenues not only in the comprehension of the physiological role of TG2 at mitochondrial level, but also about potential applications in mitochondrial-dependent diseases such as cardiovascular (e.g. ischemia/reperfusion injury) and neurodegenerative (e.g. Huntington) diseases.

\begin{abstract}
Materials and Methods
Animal husbandry and MEF generation. Original 129SvJ/C57Bl6 hybrid $\mathrm{TG}^{-1-}$ mice ${ }^{9}$ were backcrossed for 10 generations to obtain a pure C57BI6 background. All mice were kept under a $12 \mathrm{~h}$ dark/light cycle, fed standard chow ad libitum and handled in accordance with the competent Institutional Review Boards. All animals undergoing experimental procedures were littermates, individually genotyped by PCR. ${ }^{9}$ MEFs were isolated by trypsin incubation of E 14 littermate embryos from TG2 ${ }^{+1+}$ and TG2 ${ }^{-1-}$ mice. The dissociated cells were grown in DMEM medium supplemented with $10 \%$ calf serum and penicillin/ streptomycin, until we do not observe increase in cell number. After this point, the cells spontaneously resume a rapid rate of proliferation after $90-100$ days and these immortalized cell lines were used in the experiments.
\end{abstract}

Cell cultures and treatments. SK-n-BE(2) and TGA cell lines and immortalized TG2 ${ }^{+1+}$ and $\mathrm{TG} 2^{-1-}$ MEFs were cultured in RPMI supplemented with $10 \% \mathrm{FBS}$ and antibiotics. For assays cells were exposed to $5 \mathrm{mM} \mathrm{DDG}$ (Sigma) for $48 \mathrm{~h}$ or to $1 \mu \mathrm{M}$ staurosporine (STS; Sigma) for 1 or $4 \mathrm{~h}$. For experiments with 'mitochondriotropic' agents cells were treated for 30 min with $5 \mu \mathrm{M}$ of CyA (Sigma) or $30 \mu \mathrm{M}$ BA (Calbiochem) or $50 \mathrm{nM}$ ATRL (Sigma) and than exposed to apoptotic stimuli. Cells treated with CyA, BA or ATRL given alone were considered as controls. As negative control to depolarized mitochondrial membrane, cells were treated for $15 \mathrm{~min}$ at $37^{\circ} \mathrm{C}$ with $5 \mu \mathrm{M}$ carbonyl cyanide FCCP. At the end of treatment, cells were prepared for apoptosis evaluation, mitochondrial transmembrane potential analysis or immunofluorescence analysis, as stated below.

Coprecipitation assays. Whole cell extracts from low (SK-n-BE(2)) and high (TGA) TG2 expressing cells were prepared by lysis in CHAPS-IP buffer (10 mM HEPES, pH 7.4, $150 \mathrm{mM} \mathrm{NaCl}, 150 \mathrm{mM} \mathrm{KCl}, 1 \%$ CHAPS) for 30 min on ice, cleared by centrifugation for $20 \mathrm{~min}$ at $14000 \times \mathrm{g}$ at $4^{\circ} \mathrm{C}$ for $10 \mathrm{~min}$. Whole cell extracts from brains and hearts of $\mathrm{TG}^{+1+}$ mice were prepared by tissue homogenization with a Dounce homogenizer in CHAPS-IP buffer at $4^{\circ} \mathrm{C}$ and cleared by centrifugation. Protein extracts from mitochondria, isolated as described below, were performed by lysis in CHAPS-IP buffer as for WCEs. For each reaction, $500 \mu \mathrm{g}$ of total protein were preincubated with $50 \mu \mathrm{l}$ of prewashed Dynabeads-protein $\mathrm{G}$ (Invitrogen) for $1 \mathrm{~h}$ at $4^{\circ} \mathrm{C}$, with gentle shacking. Coprecipitations were performed by adding $5 \mu \mathrm{g}$ of anti-TG2 (Ab-1 or Ab-4; NeoMarkers), anti-ANT (Calbiochem) or anti-Bax (Santa Cruz) and $50 \mu \mathrm{l}$ of prewashed Dynabeads-protein G. After $4 \mathrm{~h}$ or overnight incubation, beads were recovered, washed six times with CHAPS-IP buffer. Coprecipitated proteins were detached from beads by boiling in sample buffer and separated on 4-12\% Nu-PAGE (Invitrogen), transferred to nitrocellulose and membranes were probed with anti-TG2 $(0.2 \mu \mathrm{g} / \mathrm{ml})$ and anti-ANT $(0.2 \mu \mathrm{g} / \mathrm{ml})$ antibodies for $1 \mathrm{~h}$ and then appropriate secondary horseradish peroxidaseconjugated secondary antibodies were added for $1 \mathrm{~h}$. The signals were detected with Chemiglow by means of a FluorChem SP system (Alphalnnotech).

Digitonin and alkali treatment of isolated mitochondria. Mitochondria were isolated from TG2 overexpressing cells (TGA), as described below. For digitonin treatments, mitochondria $(1 \mathrm{mg}$ of protein $/ \mathrm{ml})$ were treated with increasing amount of digitonin for $1 \mathrm{~min}$ on ice and the reaction blocked by the addition of 4 volumes of reaction buffer. Samples were centrifuged for $30 \mathrm{~min}$ at $20000 \times \mathrm{g}$ at $4{ }^{\circ} \mathrm{C}$. Supernatants, containing protein associated/inserted in the outer mitochondrial membrane or in the intramembranes space, were acetone precipitated overnight. Precipitated proteins were collected by centrifugation, washed, suspended in protein sample buffer. Pellets from the digitonin treatments, containing intact mitochondria and mitoplasts, were suspended in $50 \mathrm{mM}$ Tris-EDTA, pH 7.6 and disrupted by sonication on ice. Distribution of TG2 and ANT was assessed by western blotting comparison of the supernatants and pellets of the digitonin treatments using VDAC and Hsp60 as markers for the integrity of the mitoplasts. For alkali extraction, mitochondria were suspended ( $1 \mathrm{mg}$ of protein/ml) in freshly prepared $100 \mathrm{mM} \mathrm{Na}_{2} \mathrm{CO}_{3}(\mathrm{pH} 11.5)$ and incubated for $20 \mathrm{~min}$ on ice. $\mathrm{NaCl}(133 \mathrm{mM})$ was used as control incubation of the same ionic strength. ${ }^{22}$ The membranes were then pelleted by centrifugation. Mitochondrial membrane pellets corresponding to $10 \mu \mathrm{g}$ of proteins (the alkaliresistant fractions, pellet) and the corresponding volume of supernatants (the alkalisensitive fractions, soluble) were separated by electrophoresis on 4-12\% Nu-PAGE gel and probed with anti-ANT, VDAC, Hsp60 and TG2 antibodies.

TG2 protein substrates purification and in vitro TG2-dependent polymerization of ANT. TG2 overexpressing cells (TGA) were incubated with $5 \mathrm{mM}$ EZ-link (Pierce) for $30 \mathrm{~min}$ and then treated with $1 \mu \mathrm{M}$ staurosporine for $2 \mathrm{~h}$. Whole cell extracts (WCE) were prepared and biotinylated TG2-protein substrates purified by means of Avidin resin (Promega) following manufacturer instructions. For the in vitro polymerization of ANT, WCE from cells expressing low TG2 levels (SK-n-BE(2)) were incubated with human recombinant TG2 (hrTG2, $\mathrm{N}$-Zyme) in presence or absence of $\mathrm{Ca}^{2+}$, to activate cross-linking activity of TG2. Proteins were separated, alongside with WCEs, on 4-12\% Nu-PAGE (Invitrogen) and then probed with anti-ANT antibody.

Cell-death assays. Quantitative evaluation of apoptosis was performed by flow cytometry after double staining using FITC-conjugated Annexin V/propidium iodide (PI) apoptosis detection kit (Eppendorf), which allows discrimination between early apoptotic, late apoptotic and necrotic cell. In addition, apoptosis was also evaluated by fluorescence microscopy after cell staining with nuclear dye Hoechst 33258 (Sigma) at $37^{\circ} \mathrm{C}$ for $30 \mathrm{~min}$ as previously described. ${ }^{29}$ 
Mitochondrial membrane potential in living cells. The mitochondrial membrane potential (MMP) of controls and treated cells was studied by using JC-1 (Molecular Probes) probe. In line with this method, living cells were stained with $10 \mu \mathrm{M}$ of JC-1, as previously described. ${ }^{30}$ TMRM $(1 \mu \mathrm{M}$; Molecular Probes; red fluorescence) was also used to confirm data obtained by $\mathrm{JC}-1$ and to stain isolated mitochondria.

Preparation of isolated mitochondria. Immortalized MEFs from TG2 ${ }^{+1+}$ and $\mathrm{TG}^{-l-}$ mice were harvested by a solution containing $0.25 \%(\mathrm{w} / \mathrm{v})$ trypsin and $0.02 \%$ (w/v) EDTA, in a calcium- and magnesium-free phosphate-buffered saline solution (PBS) and collected by centrifugation. After three washings in PBS, cells were resuspended in homo-buffer $(10 \mathrm{mM}$ Hepes, $\mathrm{pH} 7.4 ; 1 \mathrm{mM}$ ethylene glycol-bis (-aminoethyl ether) $\mathrm{N}, \mathrm{N}^{\prime}, \mathrm{N}^{\prime}$-tetraacetic acid (EGTA), $0.1 \mathrm{M}$ sucrose, $5 \%$ bovine serum albumin (BSA), $1 \mathrm{mM}$ phenylmethylsulphonyl fluoride (PMSF) and complete protease inhibitor cocktail (Roche), and maintained for $10 \mathrm{~min}$ on ice. After this time, cells were homogenized with about 50 strokes of a Teflon homogenizer with B-type pestle for $10 \mathrm{~min}$ at $4^{\circ} \mathrm{C}$ to remove intact cells and nuclei, and the supernatants were further centrifuged at $10000 \times g$ at $4^{\circ} \mathrm{C}$ for $10 \mathrm{~min}$ to precipitate the heavy membrane fractions (enriched in mitochondria), as previously reported. ${ }^{31}$ These fractions were then purified by standard differential centrifugation. The mitochondrial pellet obtained was suspended in swelling buffer (SB) containing $0.1 \mathrm{M}$ sucrose, $0.5 \mathrm{M}$ sodium succinate, $50 \mathrm{mM}$ EGTA at $\mathrm{pH} 7.4,1 \mathrm{M}$ phosphoric acid (H3P04), $0.5 \mathrm{M}$ 3(N-Morpholino) butane-sulphonic acid (MOPS) and $2 \mathrm{mM}$ rotenone, kept on ice and used within $2 \mathrm{~h}$ from the preparation. Protein content in the mitochondrial preparation was determined by a spectrophotometric method using BSA as standard. The purity of mitochondria preparation was assessed by western blot checking subunit I of cytochrome $c$ oxidase (Mab; Chemicon). To obtain very purified mitochondria preparation, the main problem is commonly represented by contamination with other intracellular organelles, such as vesicles from endolysosomal compartment or Golgi apparatus. Thus, before swelling experiments, we tested the purity of our mitochondrial preparation by flow cytometry after staining with MAb specific to endolysosomal compartment or Golgi vesicle antigens, Rab5 and GM130, respectively (Santa Cruz). Samples were incubated at $4^{\circ} \mathrm{C}$ for $1 \mathrm{~h}$ and, after washing, they were labelled with anti-mouse Alexa Fluor 488 (Molecular Probes). After 45 min incubation at $4^{\circ} \mathrm{C}$, samples were washed and immediately analysed on a cytometer. As negative and positive controls we used purified mitochondria incubated with mouse IgG1 immunoglobulin or with MAb to VDAC-1 followed by an anti-mouse Alexa Fluor 488 (Figure 3c).

Swelling induction in isolated mitochondria. Mitochondria $(0.5 \mathrm{mg}$ protein $/ \mathrm{ml}$ ) were resuspended in SB at the final volume of $1.5 \mathrm{ml}$. As a general rule, the reagents under investigation were added $5 \mathrm{~min}$ after the recording was initiated. Total recording time was $25 \mathrm{~min}$. A useful positive control for these experiments consisted of the addition of $300 \mu \mathrm{M} \mathrm{Ca}^{2+}, \mathrm{a} \mathrm{Ca}^{2+}$ concentration that opens the protein transition pore. This causes amplitude swelling that is accompanied by a decrease of MMP and an increase of the outer membrane permeability, leading to the release of proteins (i.e., cytochrome c, cyt c) that are normally stored in the intramembrane space. The MMP of isolated mitochondria can be quantified by multiple methods. Here, we used a cytofluorimetric analysis after mitochondria staining with $1 \mu \mathrm{M}$ TMRM (Molecular Probes). By this method, the incorporation of dye TMRM was measured in FL3 channel: low levels of TMRM incorporation (revealed by a decrease of red fluorescence) indicated a low MMP. ${ }^{32}$

Immunofluorescence analysis. Untreated and treated cells were fixed with paraformaldehyde (4\% in PBS) and permeabilized with Triton $\times 100(0.05 \%$ in PBS) as previously described. ${ }^{31}$ Samples were then stained with MAb anti-Bax (BD Pharmingen) and PAb anti-ANT1 (Calbiochem), followed by addition of AlexaFluor595-conjugated anti-mouse IgG and AlexaFluor-488-conjugated anti-rabbit for $45 \mathrm{~min}$ at $4^{\circ} \mathrm{C}$. After washings, cells were counterstained with Hoechst 33258 and then suspended in glycerol/PBS ( $\mathrm{pH}$ 7.4). The images were acquired by intensified video microscopy (IVM) with a Nikon Microphot fluorescence microscope equipped with a Zeiss CCD camera.

Morphometric analyses. Quantitative evaluations of the Bax/ANT1 interaction were performed by analysing at least 100 cells/sample. Results are reported as percentage of cells showing yellow staining as a consequence of Bax (red) and ANT1 (green) colocalization.
Fluorescence resonance energy transfer by flow cytometry. Immortalized $\mathrm{TG}^{+1+}$ and $\mathrm{TG}^{-1-}$ MEFs were exposed to $10 \mathrm{mM}$ DDG (Sigma) for $48 \mathrm{~h}$ or to $1 \mu \mathrm{M}$ STS (Sigma) for $2 \mathrm{~h}$. After these treatments, cells were harvested, collected by centrifugation and suspended in homo-buffer. After $10 \mathrm{~min}$ of incubation, mitochondria were isolated as reported in the Materials and methods section. The mitochondria obtained by this methodology were fixed with paraformaldehyde (4\% in PBS), permeabilized with Triton $\times 100$ $(0.05 \%$ in PBS) and then stained with antibodies tagged with donor (PE) or acceptor (Cy5) dyes. Bax staining was performed using unlabelled mouse antibody (BD Pharmingen) and saturating amount of PE-labelled anti-mouse IgG (Sigma). ANT was detected by using unlabelled rabbit ANT antibody (Calbiochem) followed by Cy5-labelled anti-rabbit (BD Pharmingen). After staining, the cells were washed twice, suspended in PBS and analysed with a dual-laser FACScalibur flow cytometer (BD Biosciences). For the determination of FRET efficiency, changes in fluorescence intensities of donor-plus-acceptor-labelled cells were compared to the emission signal from cells labelled with donor- and acceptor-only fluorophores. As a further control, the cross-reactivity among all the different primary and secondary antibodies was also assessed. All data were corrected for background by subtracting the binding of the isotype controls. Efficient energy transfer resulted in an increased acceptor emission on cells stained with both donor and acceptor dyes. Statistical analysis was performed by pooling together data obtained from three independent measurements and calculating the FRET efficiency (FE) according to Riemann. ${ }^{33}$ We used the following algorithm: $F E=(F L 3 D A-F L 2 D A / a-F L 4 D A / b] /$ FL3DA, where $A$ is the acceptor and $D$ the donor, and $a=F L 2 D / F L 3 D$ and $b=\mathrm{FL} 4 \mathrm{~A} / \mathrm{FL} 3 \mathrm{~A}$

TEM studies. Cells were fixed in a mixture of $4 \%$ paraformaldehyde and $0.5 \%$ glutaraldehyde in cacodylate buffer $(0.1 \mathrm{M}, \mathrm{pH} 7.2)$ for $30 \mathrm{~min}$ at room temperature and postfixed in $0.5 \% \mathrm{OsO}_{4}$ in the same buffer for $1 \mathrm{~h}$ at room temperature. After dehydration, cells were embedded in unicryl resin. ${ }^{34}$ Serial ultra-thin sections were collected on 200-mesh gold grids. For IEM studies, thin sections were treated with PBS containing $1 \%(\mathrm{w} / \mathrm{v})$ gelatine, $1 \% \mathrm{BSA}, 5 \% \mathrm{FCS}$ and $0.05 \%$ Tween 20 and then incubated with mAb anti-TGase II (Neomarker), diluted 1: 30 in the same buffer w/o gelatine overnight at $4^{\circ} \mathrm{C}$. After washing for $1 \mathrm{~h}$ at room temperature (RT), sections were labelled with anti-mouse IgG-10 $\mathrm{nm}$ gold conjugate $(1: 10)$ for $1 \mathrm{~h}$ at RT and washed again. Negative controls were incubated with the gold conjugate alone and then counterstained with uranyl acetate and lead citrate. No non-specific gold labelling was detectable in these conditions. Both TEM and IEM samples were observed with a Philips 208 electron microscope at $80 \mathrm{kV}$.

\section{Data analysis and statistics}

All samples were analysed with a FACScan cytometer (BD Bioscience) equipped with a 488 argon laser. At least 20000 events were acquired. Data were recorded and statistically analysed by a Macintosh computer using CellQuest Software. The expression level of the analysed proteins on isolated mitochondria was expressed as a median value of the fluorescence emission curve and the statistical significance was calculated by using the non-parametric Kolmogorov-Smirnov (K/S) test. Statistical analysis of apoptosis data was performed by using Student's $t$-test or oneway variance analysis by using Statview program for Macintosh. All data reported were verified in at least three different experiments and reported as mean \pm S.D. Only $P$ values of less than 0.01 were considered as statistically significant.

Acknowledgements. We thank Vito Porcelli for skilled technical assistance. This work was supported by grants from: Prin 2006 to MP and CR; Associazione Italiana Ricerca sul Cancro to MP; Telethon (GGP06254) to MP and WM; Ministero della Sanità to WM; EU-FP6 (LSHM-CT-2004-503116) to LP.

1. Fesus $L$, Piacentini M. Transglutaminase 2 : an enigmatic enzyme with diverse functions Trends Biochem Sci 2002; 27: 534-539.

2. Knight RL, Hand D, Piacentini M, Griffin M. Characterization of the transglutaminasemediated large molecular weight polymer from rat liver; its relationship to apoptosis. Eur $\mathrm{J}$ Cell Biol 1993; 60: 210-216.

3. Lorand L, Graham RM. Transglutaminases: crosslinking enzymes with pleiotropic functions. Nat Rev Mol Cell Biol 2003; 4: 140-156.

4. Nakaoka H, Perez DM, Baek KJ, Das T, Husain A, Misono K et al. Gh: a GTP-binding protein with transglutaminase activity and receptor signaling function. Science 1994; 264: 1593-1596. 
5. lismaa SE, Wu MJ, Nanda N, Church WB, Graham RM. GTP binding and signaling by Gh/ transglutaminase II involves distinct residues in a unique GTP-binding pocket. J Biol Chem 2000; 275: 18259-18265.

6. Zhang J, Lesort M, Guttmann RP, Johnson GV. Modulation of the in situ activity of tissue transglutaminase by calcium and GTP. J Biol Chem 1998; 273: 2288-2295.

7. Hasegawa G, Suwa M, Ichikawa Y, Ohtsuka T, Kumagai S, Kikuchi M et al. A novel function of tissue-type transglutaminase: protein disulphide isomerase. Biochem $\mathrm{J} 2003$; 373 (Pt 3): 793-803.

8. Mishra S, Murphy LJ. Tissue transglutaminase has intrinsic kinase activity: identification of transglutaminase 2 as an insulin-like growth factor-binding protein-3 kinase. J Biol Chem 2004; 279: 23863-23868.

9. De Laurenzi V, Melino G. Gene disruption of tissue transglutaminase. Mol Cell Biol 2001; 21: $148-155$.

10. Falasca L, ladevaia V, Ciccosanti F, Melino G, Serafino A, Piacentini M. Transglutaminase type II is a key element in the regulation of the anti-inflammatory response elicited by apoptotic cell engulfment. J Immunol 2005; 174: 7330-7340.

11. Szondy Z, Sarang Z, Molnar P, Nemeth T, Piacentini M, Mastroberardino PG et al. Transglutaminase $2-1-$ mice reveal a phagocytosis-associated crosstalk between macrophages and apoptotic cells. Proc Natl Acad Sci USA 2003; 100: 7812-7817.

12. Bernassola F, Federici M, Corazzari M, Terrinoni A, Hribal ML, De Laurenzi V et al. Role of transglutaminase 2 in glucose tolerance: knockout mice studies and a putative mutation in a MODY patient. FASEB J 2002; 16: 1371-1378.

13. Nishikawa T, Araki E. Impact of mitochondrial ROS production in the pathogenesis of diabetes mellitus and its complications. Antioxid Redox Signal 2007; 9: 343-353.

14. Maechler $P$, Wollheim $C B$. Mitochondrial function in normal and diabetic beta-cells. Nature 2001; 414: 807-812.

15. Szondy Z, Mastroberardino PG, Varadi J, Farrace MG, Nagy N, Bak I et al. Tissue transglutaminase (TG2) protects cardiomyocytes against ischemia/reperfusion injury by regulating ATP synthesis. Cell Death Differ 2006; 13: 1827-1829.

16. Mastroberardino PG, Farrace MG, Viti I, Pavone F, Fimia GM, Melino G et al. 'Tissue' transglutaminase contributes to the formation of disulphide bridges in proteins of mitochondrial respiratory complexes. Biochim Biophys Acta 2006; 1757: 1357-1365.

17. Battaglia G, Farrace MG, Mastroberardino PG, Viti I, Fimia GM, Van Beeumen J et al. Transglutaminase 2 ablation leads to defective function of mitochondrial respiratory complex I affecting neuronal vulnerability in experimental models of extrapyramidal disorders. J Neurochem 2006; 100: 36-49.

18. Dorner A, Schultheiss HP. Adenine nucleotide translocase in the focus of cardiovascular diseases. Trends Cardiovasc Med 2007; 17: 284-290.

19. Leung AW, Halestrap AP. Recent progress in elucidating the molecular mechanism of the mitochondrial permeability transition pore. Biochim Biophys Acta 2008; 1777: 946-952.

20. Tsujimoto Y, Shimizu S. Role of the mitochondrial membrane permeability transition in cell death. Apoptosis 2007; 12: 835-840.

21. Wada F, Hasegawa H, Nakamura A, Sugimura $Y$, Kawai $Y$, Sasaki $N$ et al. Identification of substrates for transglutaminase in Physarum polycephalum, an acellular slime mold, upon cellular mechanical damage. FEBS J 2007; 274: 2766-2777.

22. Eskes R, Desagher S, Antonsson B, Martinou JC. Bid induces the oligomerization and insertion of Bax into the outer mitochondrial membrane. Mol Cell Biol 2000; 20: 929-935.

23. Ruoppolo M, Orru S, D'Amato A, Francese S, Rovero P, Marino G et al. Analysis of transglutaminase protein substrates by functional proteomics. Protein Sci 2003; 12 1290-1297.

24. Ikura K, Kita K, Fujita I, Hashimoto H, Kawabata N. Identification of amine acceptor protein substrates of transglutaminase in liver extracts: use of 5-(biotinamido) pentylamine as a probe. Arch Biochem Biophys 1998; 356: 280-286.
25. Oliverio S, Amendola A, Di Sano F, Farrace MG, Fesus L, Nemes Z et al. Tissue transglutaminase-dependent posttranslational modification of the retinoblastoma gene product in promonocytic cells undergoing apoptosis. Mol Cell Biol 1997; 17 6040-6048.

26. Matarrese P, Falzano L, Fabbri A, Gambardella L, Frank C, Geny B et al. Clostridium difficile toxin $B$ causes apoptosis in epithelial cells by thrilling mitochondria. Involvement of ATP-sensitive mitochondrial potassium channels. J Biol Chem 2007; 282 9029-9041.

27. Verrier F, Deniaud A, Lebras M, Metivier D, Kroemer G, Mignotte B et al. Dynamic evolution of the adenine nucleotide translocase interactome during chemotherapy-induced apoptosis. Oncogene 2004; 23: 8049-8064.

28. Stryer L. Fluorescence energy transfer as a spectroscopic ruler. Annu Rev Biochem 1978; 47: 819-846.

29. Straface E, Santini MT, Donelli G, Giacomoni PU, Malorni W. Vitamin E prevents UVBinduced cell blebbing and cell death in A431 epidermoid cells. Int J Radiat Biol 1995; 68 579-587.

30. Cossarizza A, Franceschi C, Monti D, Salvioli S, Bellesia E, Rivabene R et al. Protective effect of $\mathrm{N}$-acetylcysteine in tumor necrosis factor-alpha-induced apoptosis in U937 cells: the role of mitochondria. Exp Cell Res 1995; 220: 232-240.

31. Zamzami N, Maisse C, Metivier D, Kroemer G. Measurement of membrane permeability and the permeability transition of mitochondria. Methods Cell Biol 2007; 80: 327-340.

32. Rodolfo C, Falasca L, Di Giacomo G, Mastroberardino PG, Piacentini M. More than two sides of a coin? How to detect the multiple activities of type 2 transglutaminase. Methods Enzymol 2008; 442: 201-212.

33. Riemann D, Tcherkes A, Hansen GH, Wulfaenger J, Blosz T, Danielsen EM. Functional colocalization of monocytic aminopeptidase N/CD13 with the Fc gamma receptors CD32 and CD64. Biochem Biophys Res Commun 2005; 331: 1408-1412.

34. Matarrese P. Tinari A, Mormone E, Bianco GA, Toscano MA, Ascione B et al. Galectin-1 sensitizes resting human $T$ lymphocytes to Fas (CD95)-mediated cell death via mitochondrial hyperpolarization, budding, and fission. J Biol Chem 2005; 280: 6969-6985.

35. Grimm S, Brdiczka D. The permeability transition pore in cell death. Apoptosis 2007; 12 841-855.

36. Halestrap AP, Brennerb C. The adenine nucleotide translocase: a central component of the mitochondrial permeability transition pore and key player in cell death. Curr Med Chem 2003; 10: 1507-1525.

37. Vyssokikh M, Brdiczka D. VDAC and peripheral channelling complexes in health and disease. Mol Cell Biochem 2004; 256-257: 117-126.

38. Doerner A, Pauschinger M, Badorff A, Noutsias M, Giessen S, Schulze K et al. Tissuespecific transcription pattern of the adenine nucleotide translocase isoforms in humans. FEBS Lett 1997; 414: 258-262.

39. Dolce V, Scarcia P, lacopetta D, Palmieri F. A fourth ADP/ATP carrier isoform in man: identification, bacterial expression, functional characterization and tissue distribution. FEBS Lett 2005; 579: 633-637.

40. Piacentini M, Farrace MG, Piredda L, Matarrese P, Ciccosanti F, Falasca $L$ et al Transglutaminase overexpression sensitizes neuronal cell lines to apoptosis by increasing mitochondrial membrane potential and cellular oxidative stress. J Neurochem 2002; 81 1061-1072.

41. Walther T, Tschope C, Sterner-Kock A, Westermann D, Heringer-Walther S, Riad A et al. Accelerated mitochondrial adenosine diphosphate/adenosine triphosphate transport improves hypertension-induced heart disease. Circulation 2007; 115: 333-344.

42. Shen $Q$, Qin F, Gao Z, Cui J, Xiao H, Xu Z et al. Adenine nucleotide translocator cooperates with core cell death machinery to promote apoptosis in $\mathrm{C}$. Elegans. Mol Cell Biol 2009; 29: 3881-3893. 